\title{
Prevalence of anxiety disorders and symptoms in people with hearing impairment: a systematic review
}

\author{
Natalie Shoham ${ }^{1} \cdot$ Gemma Lewis $^{1} \cdot$ Graziella Favarato $^{1} \cdot$ Claudia Cooper $^{1}$ \\ Received: 9 October 2018 / Accepted: 3 December 2018 / Published online: 13 December 2018 \\ (c) The Author(s) 2018
}

\begin{abstract}
Purpose Anxiety disorders are common. Prevalence is likely to be raised in people with hearing impairment, who experience higher rates of associated risk factors. We conducted, to our knowledge, the first systematic review of the prevalence and correlates of anxiety in people with hearing impairment.

Methods We searched electronic databases and references of included studies, using predetermined criteria to retrieve original research reporting prevalence of anxiety disorders or symptoms in adult, hearing impaired populations. We assessed risk of bias using the adapted Newcastle Ottawa Scale.

Results We included 25 studies evaluating 17,135 people with hearing impairment. Community studies of higher quality reported a lifetime prevalence of anxiety disorder of $11.1 \%$ (one study) and point prevalences of $15.4-31.3 \%$ for clinically significant anxiety symptoms (five studies) in people who predominantly had acquired hearing impairment. Anxiety prevalence was higher in hearing impaired people in 8/10 studies with a comparator non-hearing impaired group. Anxiety symptoms decreased after surgical intervention for hearing in all studies investigating this. Correlates consistently associated with anxiety were tinnitus and hearing impairment severity.

Conclusions Prevalence of anxiety is higher among people with hearing impairment than the general population; our findings indicate that this excess morbidity may be related to the hearing impairment itself, as it was associated with the severity of impairment, and reduced after surgical treatment. Clinicians should be aware of the potential impact of hearing on mental health, and that where hearing ability can be improved, this may reduce anxiety.
\end{abstract}

Prospero registration number CRD42018088463.

Keywords Hearing $\cdot$ Anxiety $\cdot$ Prevalence $\cdot$ Tinnitus

\section{Introduction}

An estimated 11 million people in the UK live with significant hearing impairment [1]. The majority experience 'acquired' hearing impairment, with onset typically in older age. Around 7 per 10,000 have pre-lingual deafness, with onset before speech development. They often use sign language to communicate and some view deafness as a culture rather than as a disability $[2,3]$.

Anxiety disorders are very common. A 2004 survey of over 21,000 adults in Europe estimated the lifetime

Natalie Shoham

Natalie.shoham.16@ucl.ac.uk

1 Division of Psychiatry, University College London, 6th Floor Maple House, 149 Tottenham Court Road, London W1T 7BN, UK prevalence of any anxiety disorder at $13.6 \%$ and the 1-year prevalence at $6.4 \%$ [4]. According to ICD-10 criteria, anxiety symptoms include persistent nervousness and physical symptoms such as muscular tension, palpitations, dizziness, and epigastric discomfort [5]. Among proposed risk factors for anxiety are cognitive and functional impairments, poorer social support networks, loneliness, and traumatic events [6]. All of these risk factors are associated with hearing impairment $[2,7,8]$. Hearing impairment can impair verbal communication, increasing social exclusion and loneliness and exacerbating existing cognitive and functional impairments. It can also lead to greater dependence on others, increasing vulnerability to neglect, discrimination or abuse. It is possible that hearing impaired people may feel a greater sense of threat in challenging situations, if they are less able to understand what is happening or communicate their needs. Although risk factors for anxiety between people with 
acquired and pre-lingual hearing impairment might differ, both groups are likely to be at increased risk. Previous research has reported that rates of overall mental illness in people with hearing impairment are elevated compared with hearing populations [2, 3]. To our knowledge, although a systematic review of depression and hearing impairment is at the time of writing in progress [10], there is no systematic review investigating the prevalence and correlates of anxiety in hearing impaired people.

We aimed to assimilate evidence regarding the prevalence of anxiety disorders and clinically significant anxiety symptoms and their correlates among adults with hearing impairment.

\section{Methods}

We registered our protocol on PROSPERO on the 6th March 2018 (registration number CRD42018088463). We followed PRISMA guidelines when reporting the review.

We searched Medline, Embase, PsycINFO, and AMED databases up to 21/2/18 via Ovid interface, and CINAHL and LILACS up to $9 / 4 / 18$ for additional studies. Non-English language studies were excluded as interpreters were not available. We used the search terms: hearing or deaf* or presbycusis AND anxiety or anxious or OCD or obsessive-compulsive disorder or PTSD or post-traumatic stress disorder or agoraphobia or panic or phobia or obsess* or compuls* or phobic or agoraphobic AND rate or prevalence or risk or association or epidemiol* or incidence.

We included original research studies that reported the prevalence of anxiety symptoms or disorders, or sufficient data to calculate this, in an adult (18+) population with hearing impairment, however defined. Point, period and lifetime prevalence were accepted. We excluded studies that reported on anxiety symptoms but did not report on the number of people reaching pre-specified criteria for significant anxiety. We excluded studies that included people aged under 18 or people without sustained hearing impairment (unless there was a separate hearing impaired sample). We also excluded conference and dissertation abstracts, and studies including fewer than 30 hearing impaired people, due to the low precision of estimates based on small samples. Studies with populations with specific genetic syndromes that cause deafness were excluded due to the high risk of confounding by other syndromal symptoms or characteristics.

Where the decision to include or exclude a text was not clear, we resolved this by discussion. One-tenth of the study abstracts identified by NS from the original search were cross-checked by a co-author (CC), who did not identify any additional eligible papers. Reference lists of eligible studies were then hand-searched for additional studies.

\section{PRISMA flowchart: see Fig. 1}

Data were extracted by NS. Where sample size or anxiety prevalence as a percentage was not given, we calculated this from the available data. Where hearing impairment was divided into subgroups with differing levels of impairment, results from these groups were pooled. Some hearing impaired groups within studies were not pooled however; for example, where it was felt useful to retain a subgroup because it reported on patients with a comorbid condition. We synthesised findings and presented these as a narrative account. We decided a priori that we would meta-analyse where outcomes and populations from three or more studies were considered to be sufficiently homogeneous, by using the metan command in STATA to obtain a pooled prevalence [11]. Confidence intervals were generated using an online calculator [12]. We also identified correlates of anxiety in the studies, which we defined as measured variables which were associated with anxiety in hearing impaired populations.

\section{Study quality}

The quality of studies was assessed independently by $\mathrm{CC}$ and NS using the Newcastle-Ottawa Quality Rating Scale (NOS) adapted for cross-sectional studies [13]. We discussed any discrepancies in ratings to reach a consensus. This was a deviation from protocol as the NOS was found to be more suited to the purpose than the STROBE guidelines, which are intended to critically appraise studies and does not give a quality score [14]. The NOS assesses quality based on sample selection, comparability between groups, and outcome assessment. Up to five stars are awarded for selection, based on how representative the sample is of the target population, whether justification is given for the sample size, whether comparability between respondents and non-respondents is established, and whether the exposure was measured using a validated tool. Comparability concerns whether confounders have been controlled for between exposure groups and is worth two stars. Up to three stars are awarded for outcome being measured by independent blind assessment or record linkage or self-report, and an appropriate statistical test being used. As there is no agreed scoring method for the NOS as applied to prevalence studies, we judged studies to be of higher quality if the following criteria were met: sample selection was either (a) truly representative of the average in the target population (all subjects or random sampling) or (b) somewhat representative of the average in the target population (non-random sampling); and measurement of the outcome was clearly described (ideally using 


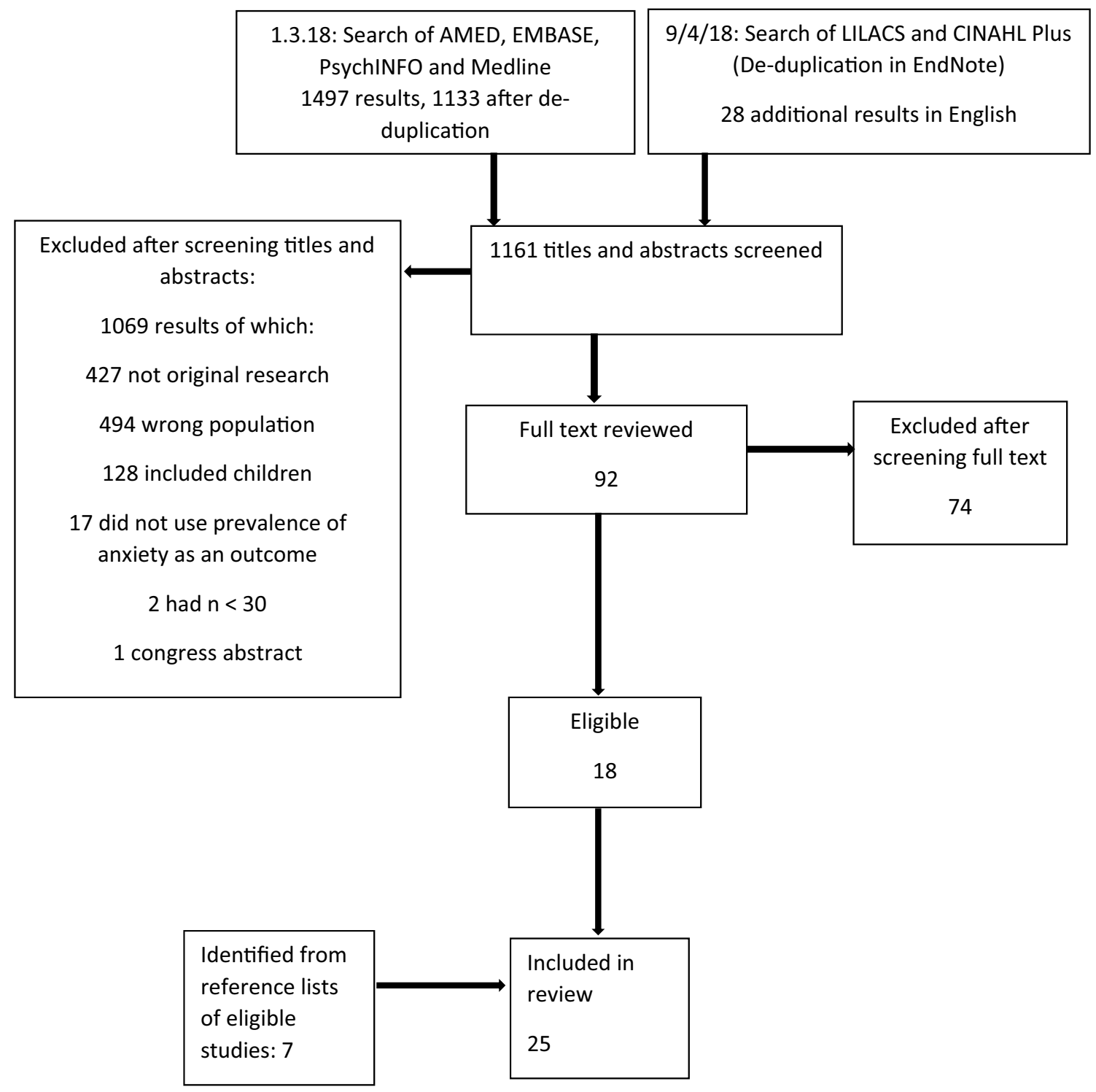

Fig. 1 Prisma flowchart

independent blind assessment or record linkage). We prioritised studies that were rated as higher quality in our narrative synthesis.

\section{Results}

Figure 1 illustrates our search strategy results. We included 25 studies. Most $(n=20)$ took place in Europe or the USA, and most $(n=21)$ were published in the last 20 years. 19 of the studies were of higher quality according to our criteria. 17,135 people with hearing impairment were included, with a reported age range from 18 to 101 years. Presence of hearing impairment was variably determined by self-report, ENT assessment, inclusion on deaf registers and inclusion in specialist services for the deaf. 15 studies included a separate comparator group.

We did not meta-analyse data, as the broad range of outcome measures used precluded this. We illustrated results with a forest plot, created using STATA version 15.1 [11] (see Fig. 2). Results are narratively synthesised below. We made a post hoc decision to group studies according to the setting, as populations within in ear nose and throat (ENT) and within mental health settings appeared to be broadly similar.

\section{Anxiety measures}

In six studies, anxiety disorder presence was evaluated using diagnostic interviews. These were the Composite 


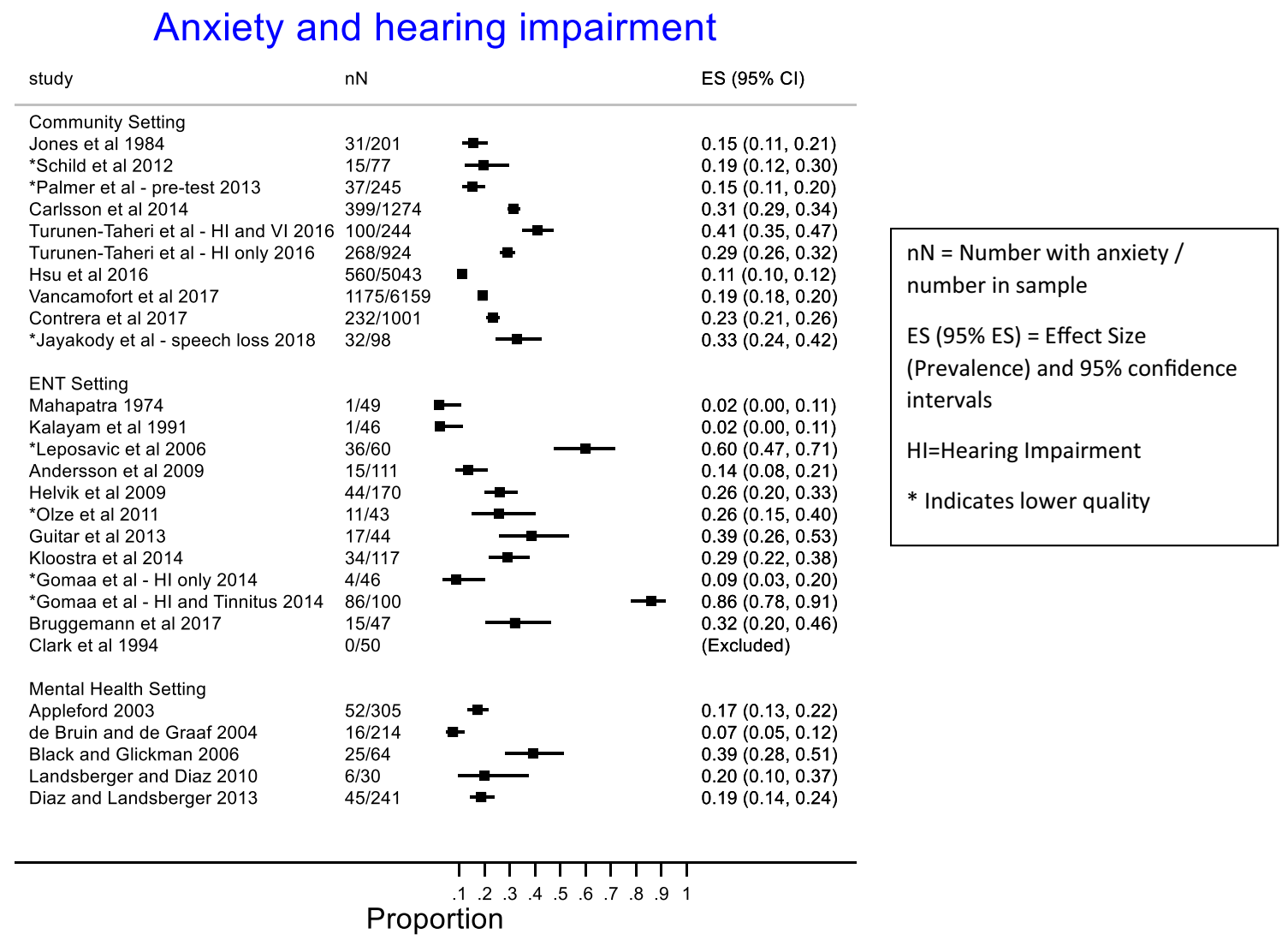

Fig. 2 Forest plot of prevalence of anxiety disorder/symptoms in included studies

International Diagnostic Interview (CIDI) [15], the Clinician Administered Post-Traumatic Stress Disorder Scale (CAPS) [8], the Stanford Panic Inventory [16], and unspecified standardised interviews based on the Diagnostic and Statistical Manual (DSM) or International Classification of Diseases (ICD) [17-19]. Six studies reported on clinical diagnoses of anxiety disorder, using accepted diagnostic criteria (ICD or DSM) from medical notes [9, 20-24].

Thirteen studies used measures of anxiety symptoms, two of which overlapped with the studies reporting diagnoses $[16,18]$. Four of the studies reporting symptoms used the Hospital Anxiety and Depression Scale-Anxiety (HADSA); two with a cut-point for clinical significance of 8 or greater $[25,26]$ and two with a cut-point of 10 or greater $[27,28]$. These cut-points are validated as indicative of mild and severe anxiety, respectively [29]. Three studies used the Depression, Anxiety and Stress Scale (DASS), with a cutpoint of 8 or greater in line with the scoring guidance [30, 31], or not stated [32]. One study used selected questions from the Delusion-Symptoms States Inventory with a cutoff of 6, stating that this was in accordance with psychiatric opinion [33]. One used the Generalised Anxiety Disorder-7 scale (GAD-7) with a validated cut-off of 10 or greater [34]. Another used the Spielberger State-Trait Anxiety
Inventory (STAI-S) with a commonly agreed cut-point of 40 or greater [35]. An abbreviated version of the Hopkins Symptom checklist was used by one study, with a positive response to 2 symptoms or strongly positive response to 1 symptom indicating anxiety presence; a validated approach [36]. Another study used a single validated question about anxiety from the Perceived Health Questionnaire (PHQ): During the last two weeks, have you felt troubled by anxiety? [37]. Another used a single question: Overall in the past 30 days, how much of a problem did you have with worry or anxiety? [38] — with response alternatives: none, mild, moderate, severe, and extreme. Those who answered severe and extreme were classified as having anxiety; the approach taken by the World Health Survey. The remaining study used the Minnesota Multiphasic Personality Inventory (MMPI) in addition to psychiatric diagnosis, but did not explain how the MMPI scores were linked to diagnosis [18].

\section{Community studies (Table 1)}

Nine of the studies recruited participants from the community settings. One reported lifetime prevalence, and the others point prevalence. 


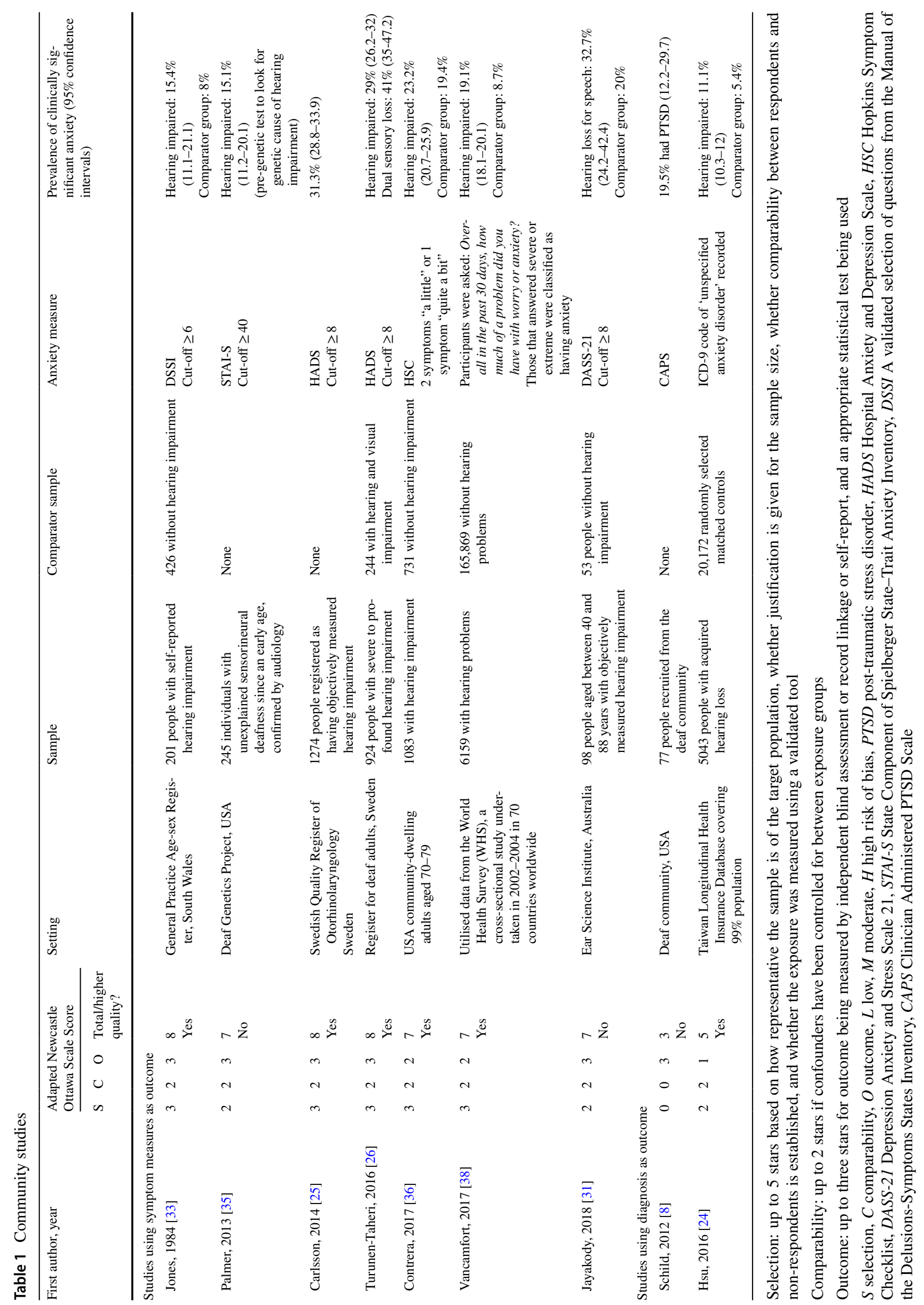




\section{Studies reporting diagnoses}

Two community studies reported prevalence of anxiety disorder diagnoses. A very large study reported the prevalence of an archival ICD-9 diagnosis in a sample of 5043 hearing-impaired people and 20,172 matched controls from a Taiwanese insurance database. It found an $11.1 \%$ lifetime prevalence of clinically diagnosed 'unspecified anxiety disorder' in the hearing impaired group compared to $5.4 \%$ in the controls $(p$ value $<0.001)$ [24]. Specific anxiety disorder diagnoses were not considered by this study.

The other study reported that the prevalence of PTSD among 77 culturally Deaf people recruited from the Deaf community in the USA. The prevalence was $19.5 \%$ [8]. This study recruited a convenience sample, so was rated lower quality due to the potential for selection bias. Participants in this study had been deaf from childhood. All had a history of traumatic events.

\section{Studies reporting symptoms}

The other six community studies measured prevalence of anxiety symptoms. They collectively assessed 10,228 hearing impaired people and 167,079 people in comparator groups. Four were of higher quality and took place in the USA, Sweden, Wales, and in Belgium using a global sample. They predominantly recruited people with acquired hearing loss and the reported prevalence of significant anxiety symptoms ranged from 15.4 to $31.3 \%$ [25, 33, 36, 38]. The study using the global (World Health Survey) sample included 6,159 randomly selected people with self-reported hearing impairment and 165,869 controls across 42 countries making it by far the largest study [38]. It assessed presence of anxiety using a single self-report question. It found a prevalence of $19.1 \%$ in the hearing impaired people compared to $8.7 \%$ in the non-hearing impaired sample, and following adjustment gave an odds ratio of anxiety presence in hearing impairment of 1.50 [95\% CI 1.29-1.74].

Another study recruited people from the Swedish register for deaf adults and found a prevalence of significant anxiety symptoms of $41 \%$ in people with dual (visual and hearing) sensory loss, and $29 \%$ in people with only hearing impairment [26].

Two studies were of lower quality due to relying on selfselection of participants $[31,35]$. One of these notably investigated people with early-onset deafness who were awaiting testing for genetic causes of deafness in the USA and reported a $15.1 \%$ prevalence of significant anxiety symptoms [35]. The other looked at 98 older adults with hearing loss for speech and found a $32.7 \%$ prevalence of anxiety symptoms [31]. Every study that compared hearing impaired and non-hearing impaired people found a statistically significant higher prevalence of anxiety disorder or symptoms in the hearing impaired group [31, 33, 36, 38].

\section{Summary}

- In people with hearing impairment recruited from community samples, one large study reported an $11.1 \%$ lifetime prevalence of 'unspecified anxiety disorder'.

- A global study with a very large sample reported a prevalence of self-reported anxiety over the past month of $19.1 \%$ in the hearing impaired sample.

- Significant anxiety symptom prevalences of $15.4-31.3 \%$ were reported in five studies in people with predominantly acquired hearing impairment.

- One study reported a $41 \%$ prevalence of significant anxiety symptoms in people with dual sensory loss.

- Every study that compared hearing impaired and nonhearing impaired people found a higher prevalence of anxiety disorder or symptoms in the hearing impaired group.

\section{Studies in ENT settings (Table 2)}

We included 11 studies that recruited participants from ear nose and throat (ENT) hospital settings and reported point prevalence. Most of the 898 participants included in these studies had acquired hearing impairment and sample mean ages ranged from 45 to 70 years. The majority of these studies reported an objective method of identifying hearing impairment including audiometry $[17,19,27,37]$ and speech recognition scores $[16,17,27,34]$, though the exact methods and thresholds used varied between studies.

\section{Studies reporting diagnoses}

Five studies assessed the prevalence of anxiety disorder diagnoses in a total of 252 hearing impaired people. Three of these were over 20 years old: one from UK ENT inpatients reported a $4.6 \%$ prevalence of 'anxiety state' based on psychiatric interview in 1974 [19], and one reported an anxiety disorder prevalence of $2.2 \%$ in USA outpatients in 1991 [17]. The other older study reported panic disorder prevalence in USA outpatients in 1994. It compared people presenting with hearing impairment, of whom none had panic disorder, and people presenting with dizziness, of whom $20 \%$ had panic disorder [16].

Two studies were more recent; conducted in 2006 and 2017. In one, outpatients awaiting cochlear implantation in Germany had a $39.9 \%$ prevalence of anxiety disorder [15]. The other study was of lower quality due to choosing a highly select group of patients, but reported an anxiety disorder rate of $60 \%$ in people presenting to an ENT outpatient clinic in Serbia for stapedectomy [18]. 


\section{Studies reporting symptoms}

Six ENT studies collectively assessed 646 hearing-impaired people and measured the prevalence of anxiety symptoms. The participants were people presenting for cochlear implantation in ENT outpatient clinics in Germany, The Netherlands, New Zealand, and Sweden [27, 28, 32], and in one case for audiological rehabilitation in Norway [37]. One study reported anxiety symptom prevalence in groups with hearing loss for speech or high-frequency sounds separately, with some overlap between groups [31]. To avoid counting patients twice, only the loss for speech group's results have been pooled across severity domains and reported in our review. The proportion scoring above the study-defined cut-points for anxiety symptoms across the studies of higher quality ranged from 13.5 to $38.6 \%$ [27, 28, 32, 37].

One study was deemed lower quality due to not clearly reporting the method of sample selection. It took place in an Egyptian ENT outpatient clinic and reported the prevalence of clinically significant anxiety symptoms to be $8.7 \%$ in people with hearing impairment without tinnitus and $86 \%$ in those with hearing impairment and tinnitus [30].

\section{Pre- and post-intervention ENT studies}

Five of the above studies sampled patient groups before and at least 6 months after surgical intervention (cochlear implantation or stapedectomy) to improve acquired hearing loss [15, 18, 28, 32, 34]. All but one [32] examined the same group longitudinally. Two were of lower quality due to unclear reporting of sample selection [18, 34]. Significantly fewer patients had anxiety symptoms in post-intervention groups compared to pre-intervention groups across all five studies ( $p$ values from $<0.001$ to $<0.05$ ). The absolute reduction in prevalence in post-intervention groups ranged from 1.9 to $30 \%$.

\section{Summary}

- Across studies of higher quality, the reported anxiety prevalence in hearing impaired populations ranged from 2.2 to $31.9 \%$ for any anxiety disorder, and $13.5-38.6 \%$ for presence of clinically significant anxiety symptoms.

- Anxiety symptoms were lower (by between 1.9 and 30\%) after surgical intervention to improve hearing in all five studies assessing this.

\section{Studies in mental health settings (Table 3)}

Four studies assessed a period prevalence of diagnoses of any mental disorder in Deaf mental health services, which primarily treat pre-lingually and culturally Deaf adults [22]. All were of higher quality. The reported prevalence of anxiety disorder diagnoses ranged from 7.5 to $39.1 \%$. Two studies from a USA inpatient unit and combined UK inpatient and outpatient services found the prevalence to be higher in deaf compared with hearing samples [9, 23]. One study reported an anxiety disorder prevalence of $18.7 \%$ in a USA outpatient unit and was the only study to report a prevalence of anxiety disorders that was higher in hearing than deaf patients [20]. This may be because anxiety is commonly comorbid with other psychiatric disorders, so the comparator group also had elevated rates. The remaining study took place in a Netherlands' outpatient unit and had no comparator group [22]. This found an anxiety disorder prevalence of $7.5 \%$.

One study of higher quality recruited hearing impaired people from generic mental health services. It reported anxiety disorder prevalence of $20 \%$ in 30 hearing impaired people and 18\% in 60 non-hearing impaired people [21].

\section{Summary}

- The reported prevalence of anxiety disorder in hearing impaired mental health patients ranged from 7.5 to $39.1 \%$.

\section{Correlates of anxiety symptoms}

\section{Tinnitus}

Six studies explored the association between anxiety and tinnitus in hearing impaired populations. Five were in ENT settings and of these, two were of lower quality [30,34]. The other was a community study [25]. Tinnitus severity was measured by various methods of self-report, including the Tinnitus Handicap Inventory, Tinnitus Questionnaire, and Tinnitus Handicap Questionnaire. Three of the studies found that people with hearing loss and tinnitus reported higher levels of anxiety than people with hearing loss only [15, 25, 34]. One reported a reversal of post-intervention improvements in depression and anxiety for patients who developed tinnitus [28]. Of the lower quality studies, one also found that longer tinnitus symptom duration, but not severity, was associated with higher rates of anxiety symptoms [30]. The other found that greater tinnitus handicap was significantly correlated with higher anxiety symptom score, following cochlear implantation but not prior to it [34].

\section{Other factors linked to sensory impairment}

Three studies assessed the relationship between hearing impairment severity and anxiety and all found a positive association [30, 31, 36]. Severity of hearing impairment was universally measured by audiometry. One study also noted an association between greater anxiety and longer 


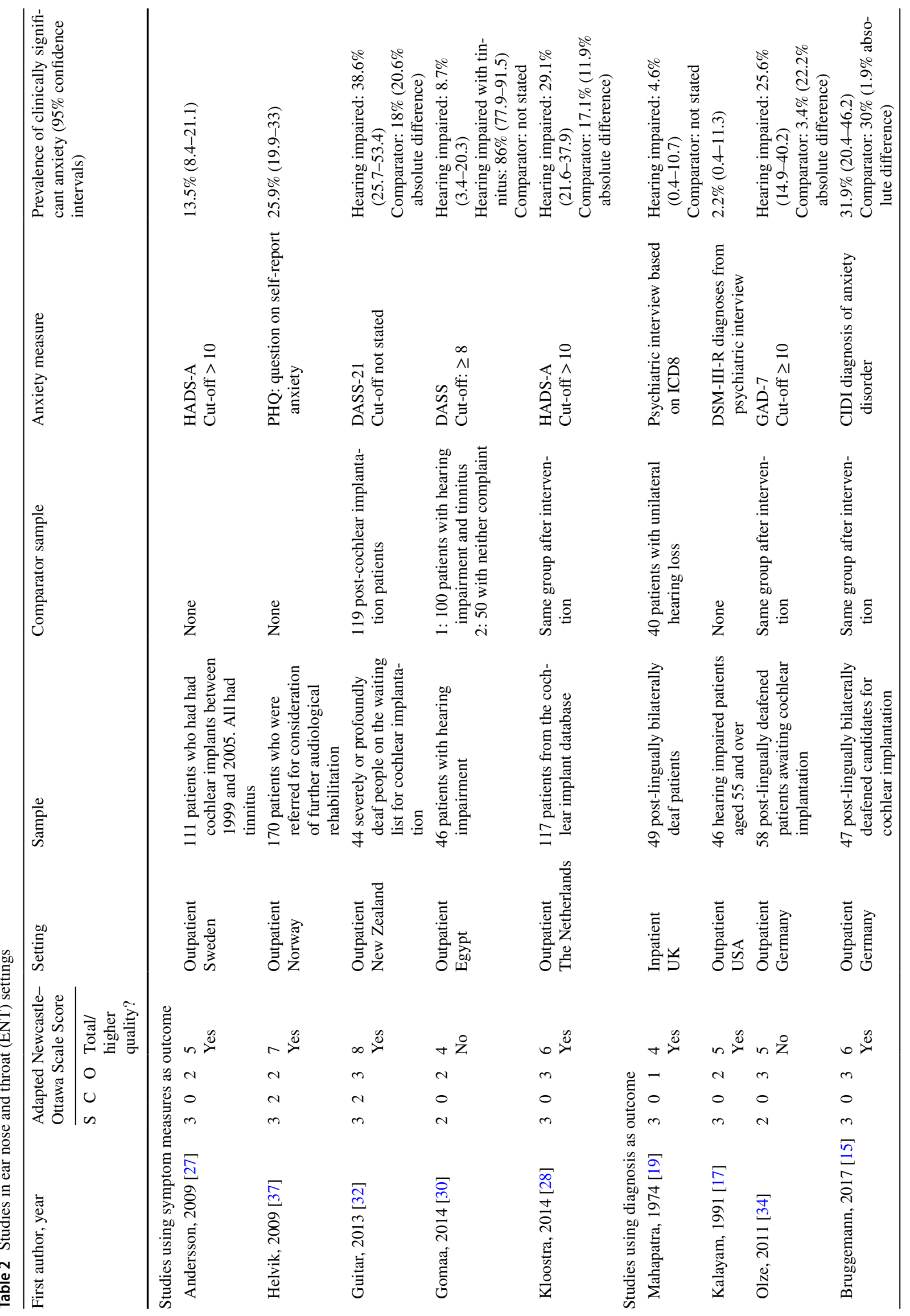




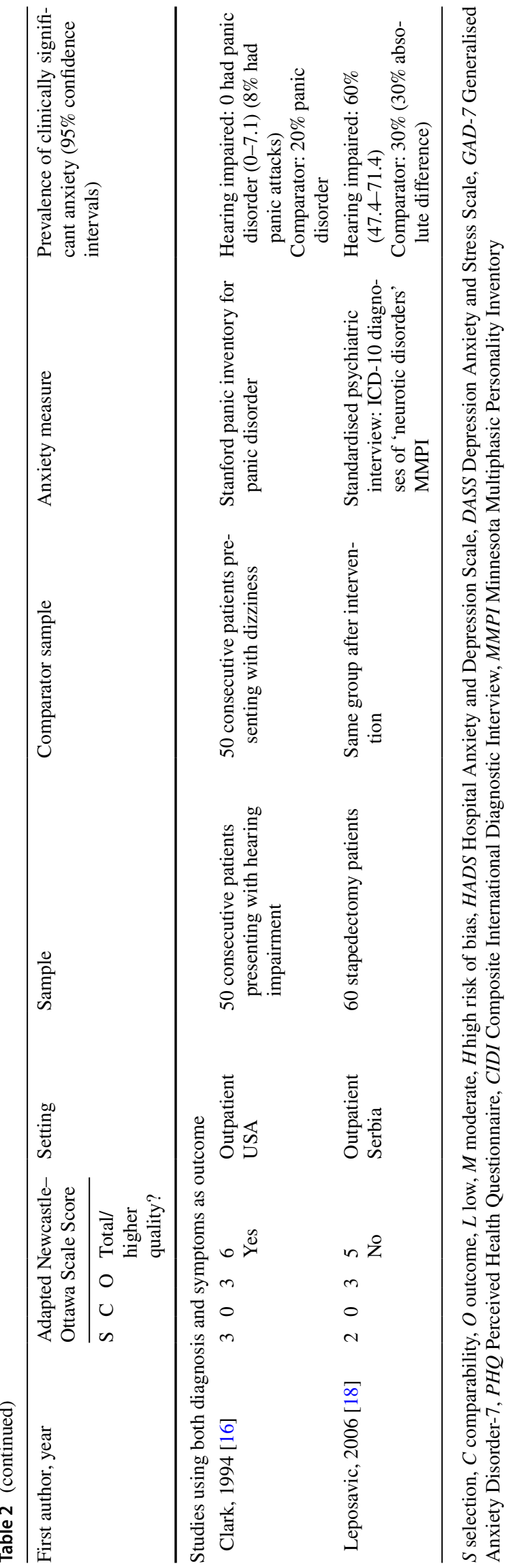

hearing impairment duration [30], but another did not find this [34]. The presence of visual impairment and vertigo also correlated with statistically higher anxiety levels [25, 26]. Finally, the odds of reporting anxiety were found to increase significantly with increasing number of physical comorbidities [38].

\section{Psychosocial factors}

Higher disease-related quality-of-life score was associated with lower anxiety scores in two studies [15, 34]. In other studies, better communication and adaptation to loss [18]; active behaviour and the establishment of social interactions; and active stress management all associated with lower anxiety following surgery [15].

\section{Other factors}

Although several of the studies looked for an association between anxiety and gender, statistical evidence of an association was not found [27, 30, 35, 36].

\section{Summary}

- Tinnitus was consistently associated with presence of anxiety in hearing impaired people in 6/6 studies examining this.

- Severity of hearing impairment was also identified as a correlate of anxiety in $3 / 3$ studies examining this.

\section{Discussion}

\section{Main findings}

Anxiety prevalence was consistently higher in people with hearing impairment than comparison groups without hearing impairment. Of the studies that reported anxiety prevalence in a comparator group, the prevalence was higher in the hearing impaired group in 8 studies $[9,21,23,24,31$, $33,36,38]$. This was not the case when a comparator group presenting with dizziness was assessed for panic disorder, which is perhaps unsurprising since dizziness is a symptom of panic disorder [16]. There was just one exception to this where the comparator group had no reported physical comorbidity, though this group was selected from a psychiatric population [20]. Anxiety is a common comorbidity with most other psychiatric conditions, which may explain this. Post-operative anxiety prevalences were lower than pre-operative prevalences in all five studies evaluating this in populations receiving surgical interventions to enhance 
Table 3 Studies from specialist mental health services

\begin{tabular}{|c|c|c|c|c|c|c|c|c|c|}
\hline \multirow[t]{2}{*}{ Author, year } & \multicolumn{4}{|c|}{$\begin{array}{l}\text { Adapted Newcastle- } \\
\text { Ottawa Scale Score }\end{array}$} & \multirow[t]{2}{*}{$\begin{array}{l}\text { Setting and } \\
\text { country }\end{array}$} & \multirow[t]{2}{*}{ Sample } & \multirow[t]{2}{*}{$\begin{array}{l}\text { Comparator } \\
\text { sample }\end{array}$} & \multirow[t]{2}{*}{$\begin{array}{l}\text { Anxiety disorder } \\
\text { measure }\end{array}$} & \multirow{2}{*}{$\begin{array}{l}\text { Prevalence of } \\
\text { anxiety disorder } \\
\text { (95\% confidence } \\
\text { intervals) }\end{array}$} \\
\hline & $\mathrm{S}$ & $\mathrm{C}$ & $\mathrm{O}$ & $\begin{array}{l}\text { Total/ } \\
\text { higher } \\
\text { quality? }\end{array}$ & & & & & \\
\hline $\begin{array}{l}\text { Appleford, } 2003 \\
\text { [23] }\end{array}$ & 2 & 0 & 2 & $\begin{array}{l}4 \\
\text { Yes }\end{array}$ & $\begin{array}{l}\text { Inpatient and } \\
\text { Outpatient Deaf } \\
\text { Mental Health } \\
\text { Service } \\
\text { UK }\end{array}$ & $\begin{array}{l}305 \text { hearing } \\
\text { impaired } \\
\text { patients }\end{array}$ & $\begin{array}{l}621 \text { hearing } \\
\text { patients from } \\
\text { same hospital or } \\
\text { clinic }\end{array}$ & $\begin{array}{l}\text { ICD-10 } \\
\text { diagnoses }^{\mathrm{a}}\end{array}$ & $\begin{array}{l}17 \%(13.3-21.7) \\
8 \% * *\end{array}$ \\
\hline $\begin{array}{l}\text { De Bruin, } 2004 \\
\text { [22] }\end{array}$ & 2 & 0 & 2 & $\begin{array}{l}4 \\
\text { Yes }\end{array}$ & $\begin{array}{l}\text { Outpatient Deaf } \\
\text { Mental Health } \\
\text { Service } \\
\text { The Netherlands }\end{array}$ & 214 outpatients & None & $\begin{array}{l}\text { DSM IV } \\
\text { diagnoses }^{\mathrm{a}}\end{array}$ & $7.5 \%(4.7-11.8)$ \\
\hline Black, 2006 [9] & 2 & 0 & 2 & $\begin{array}{l}4 \\
\text { Yes }\end{array}$ & $\begin{array}{l}\text { Inpatient Deaf } \\
\text { Mental Health } \\
\text { Service } \\
\text { USA }\end{array}$ & $\begin{array}{l}64 \text { hearing } \\
\text { impaired } \\
\text { patients }\end{array}$ & $\begin{array}{l}180 \text { hearing } \\
\text { patients from } \\
\text { same hospital }\end{array}$ & $\begin{array}{l}\text { DSM IV } \\
\text { diagnoses }^{\mathrm{a}}\end{array}$ & $\begin{array}{l}39.1 \%(28.1-51.3) \\
8.8 \% * *\end{array}$ \\
\hline $\begin{array}{l}\text { Landsberger, } 2010 \\
\text { [21] }\end{array}$ & 2 & 0 & 2 & $\begin{array}{l}4 \\
\text { Yes }\end{array}$ & $\begin{array}{l}\text { Inpatient Mental } \\
\text { Health Service } \\
\text { USA }\end{array}$ & 30 deaf patients & $\begin{array}{l}60 \text { hearing } \\
\text { patients from } \\
\text { same hospital }\end{array}$ & $\begin{array}{r}\text { DSM IV-TR } \\
\text { diagnoses }^{\mathrm{a}}\end{array}$ & \\
\hline Diaz, 2013 [20] & 2 & 1 & 2 & $\begin{array}{l}5 \\
\text { Yes }\end{array}$ & $\begin{array}{l}\text { Outpatient Deaf } \\
\text { Mental Health } \\
\text { Service } \\
\text { USA }\end{array}$ & 241 deaf patients & $\begin{array}{l}345 \text { hearing } \\
\text { patients from } \\
\text { same clinic }\end{array}$ & $\begin{array}{l}\text { DSM IV } \\
\text { diagnoses }^{\mathrm{a}}\end{array}$ & $\begin{array}{l}18.7 \%(14.3-24.1) \\
30.1 \% * *\end{array}$ \\
\hline
\end{tabular}

$S$ selection, $C$ comparability, $O$ outcome, $L$ low, $M$ moderate, $H$ high risk of bias, $P T S D$ post-traumatic stress disorder

${ }^{\mathrm{a}}$ From medical records

**Comparator Group Selected from same hospital or clinic

hearing. People with hearing impairment were more likely to have clinically significant anxiety if they also experienced tinnitus [15, 25, 27, 28, 30, 34] and if their hearing impairment was more severe [30, 31, 36].

Overall, our findings suggest that anxiety disorders and anxiety symptoms are likely to be higher in people with hearing impairment than the general population. The findings that anxiety was related to hearing severity and that it decreased after surgical interventions to improve hearing are possible indications that the excess anxiety found in hearing impaired population could result from the impairment itself rather than other influences, though this could not be assessed in this study.

\section{Limitations}

The studies included used different measures of anxiety, making it difficult to compare results across them. Some of the anxiety measures were not, to our knowledge, validated in a hearing impaired population. This might be particularly important when considering participants whose first language is not a spoken language. Some studies measured anxiety through a small number of self-report questions [26, 36], which may have been less reliable or valid than longer interview schedules. In the mental health service-based studies there was potential for cultural misunderstanding and language barriers to increase the risk of misdiagnosis among the deaf. For example, the grammar structure of British sign language could lead clinicians to wrongly conclude that a patient is thought-disordered based on their written communication [39].

A number of studies did not describe the method of sample selection. Recent studies reported a higher prevalence of anxiety disorder based on standardised psychiatric interview than older studies, suggesting that diagnostic practices may have changed over time. This may also be because general population awareness of anxiety has increased. The studies included were heterogeneous in terms of how hearing impairment was measured; whether the hearing impairment was acquired pre- or post-lingually; recruitment source (clinical or non-clinical); and presence of additional conditions such as tinnitus [25, 27, 28, 30], vertigo [25] or deafblindness [26]. The variety of study settings may also be a strength of this review, as it captures a broad spectrum of 
the hearing impaired population across countries and age ranges.

The studies based in mental health services reported routinely collected clinical data, so the procedures for making diagnoses may not have been standardised across services. This also introduced the possibility of diagnostic overshadowing, in which diagnosing a 'major' mental disorder such as psychosis might have been prioritised in a specialist service over assessment for common mental illness. The authors of the mental-health service-based study which found a lower anxiety prevalence in deaf people suggest that perhaps D/deaf people in their region had to be more severely unwell than hearing people to receive professional support, supporting diagnostic overshadowing as a potential explanation for the discrepant results compared to similar studies. Although it is tempting to infer from ENT studies that an improvement in hearing led to an improvement in anxiety, anxiety about the procedure itself may have led to the post-procedure reduction in anxiety. Consistent with this is the finding by one study that time on the waiting list was associated with stress [32].

We did not include non-English language studies and unpublished studies in the review. We excluded people under the age of 18 , to ensure that findings were relevant to people referred to adult mental health services. Since our primary aim was to investigate prevalence, we only assessed correlates of hearing impairment and anxiety in studies that also reported a prevalence. We have not considered depression, which is linked to anxiety. In fact it has recently been argued that generalised anxiety disorder is not a separate construct from depression [40]. Even with this debate, we would argue that it remains useful to look at common mental illness from the perspective of anxiety.

The Newcastle-Ottawa Scale (NOS) is not originally designed to be used with prevalence studies, and so we have had to adapt it to assess the quality of studies for this review and this approach is not validated.

A further limitation of our review is the scope of the question, which related only to prevalence and correlates. Hence no inference about the cause of heightened anxiety prevalence in hearing impaired people can be drawn from these results.

\section{Implications}

Based on these results, it appears that the prevalence of clinically significant anxiety in people with hearing impairment is higher than for people without hearing impairment. Healthcare professionals should be alert to this, and have a low threshold for enquiring about anxiety when seeing people with hearing impairment, especially those with tinnitus or severe impairment. This should include making adaptations to communication where appropriate, such as through the use of hearing loops or sign language interpreters. Anxiety is known to be a treatable with both psychological therapies such as cognitive behavioural therapies (CBT) and medications [41]. The finding by one study that active behaviour and active stress management reduced anxiety following surgery suggests that CBT or behavioural therapy has potential to be effective [15]. The provision of mental health facilities competent in deaf culture for the pre-lingually Deaf population is also a key part of addressing the burden of anxiety disorders.

The mechanisms that might lead to increased prevalence of clinically significant anxiety in people with hearing impairment are not well-understood. Further research into the nature of the association is needed, ideally using longitudinal cohorts. Social functioning, discrimination and abuse might mediate the relationship between hearing impairment and anxiety, and difficulty accessing mental health services might mean that people with hearing impairment live with anxiety disorders for longer.

In conclusion, the literature shows a considerable burden of anxiety symptoms and disorders in people with hearing impairment, and the prevalence appears to be higher than for people without hearing impairment. This review highlights the importance of making mental health services accessible for hearing impaired people and further investigating the nature of the association between hearing impairment and anxiety.

Open Access This article is distributed under the terms of the Creative Commons Attribution 4.0 International License (http://creativeco mmons.org/licenses/by/4.0/), which permits unrestricted use, distribution, and reproduction in any medium, provided you give appropriate credit to the original author(s) and the source, provide a link to the Creative Commons license, and indicate if changes were made.

\section{References}

1. Action on Hearing Loss. Facts and Figures-Webpage. 24.1.18 [cited 2018 24.1.18]; https://www.actiononhearingloss.org.uk/ about-us/our-research-and-evidence/facts-and-figures/. Accessed Jan 2018

2. Fellinger J, Holzinger D, Pollard R (2012) Mental health of deaf people. Lancet 379(9820):1037-1044

3. Øhre B, von Tetzchner S, Falkum E (2011) Deaf adults and mental health: a review of recent research on the prevalence and distribution of psychiatric symptoms and disorders in the prelingually deaf adult population. Int J Ment Health Deafness 1(1):3-22

4. Investigators EM et al (2004) Prevalence of mental disorders in Europe: results from the European Study of the Epidemiology of Mental Disorders (ESEMeD) project. Acta Psychiatr Scand 109:21-27

5. World Health Organisation. Generalized Anxiety Disorder-ICD10 Criteria. http://apps.who.int/classifications/icd10/browse/2016/ en\#/F41.1. Accessed July 2018 
6. Vink D, Aartsen MJ, Schoevers RA (2008) Risk factors for anxiety and depression in the elderly: a review. J Affect Disord 106(1):29-44

7. Action on Hearing Loss. Hearing Matters-Report. Available at: http://www.actiononhearingloss.org.uk/how-we-help/informatio $\mathrm{n}$-and-resources/publications/research-reports/hearing-mattersreport/. Accessed Dec 2018

8. Schild S, Dalenberg CJ (2012) Trauma exposure and traumatic symptoms in deaf adults. Psychol Trauma Theory Res Pract Policy 4(1):117-127

9. Black PA, Glickman NS (2006) Demographics, psychiatric diagnoses, and other characteristics of North American deaf and hard-of-hearing inpatients. J Deaf Stud Deaf Educ 11(3):303-321

10. Lawrence B, Jayakody D, Friedland P (2018) Depression and age-related hearing loss in older adults: a systematic review and meta-analysis. PROSPERO 2018 CRD42018084494. https ://www.crd.york.ac.uk/prospero/display_record.php?Recor $\mathrm{dID}=84494$. Accessed Oct 2018

11. StataCorp LP (2015) Stata statistical software: release 14. Stata Corp, College Station

12. Lowry R (2001-2018) The confidence interval of a proportion. [cited 2018 30.4.18]; http://www.vassarstats.net/prop1.html. Accessed Nov 2018

13. Modesti PA et al (2016) Panethnic differences in blood pressure in Europe: a systematic review and meta-analysis. PLoS One 11(1):e0147601

14. Von Elm E et al (2007) The strengthening the reporting of observational studies in epidemiology (STROBE) statement: guidelines for reporting observational studies. PLoS Med 4(10):e296

15. Bruggemann $P$ et al (2017) In patients undergoing cochlear implantation, psychological burden affects tinnitus and the overall outcome of auditory rehabilitation. Front Hum Neurosci 11:226

16. Clark DB et al (1994) Panic in otolaryngology patients presenting with dizziness or hearing loss. Am J Psychiatry 151(8):1223-1225

17. Kalayam B et al (1991) Patterns of hearing loss and psychiatric morbidity in elderly patients attending a hearing clinic. Int J Geriatr Psychiatry 6(3):131-136

18. Leposavic L et al (2006) Psychosocial aspects of acquired hearing impairment in the patients with otosclerosis. Psychiatr Danubina 18(1-2):30-38

19. Mahapatra S (1974) Deafness and mental health: psychiatric and psychosomatic illness in the deaf. Acta Psychiatr Scand 50(6):596-611

20. Diaz DR et al (2013) Psychiatric disorder prevalence among deaf and hard-of-hearing outpatients. Compr Psychiatry 54(7):991-995

21. Landsberger SA, Diaz DR (2010) Inpatient psychiatric treatment of deaf adults: demographic and diagnostic comparisons with hearing inpatients. Psychiatr Serv 61(2):196-199

22. de Bruin E, de Graaf R (2004) What do we know about deaf clients after thirteen years of ambulatory mental health care? An analysis of the PsyDoN database, 1987-1999. Am Ann Deaf 149(5):384-393
23. Appleford J (2003) Clinical activity within a specialist mental health service for deaf people: comparison with a general psychiatric service. Psychiatrist 27(10):375-377

24. Hsu WT et al (2016) Increased risk of depression in patients with acquired sensory hearing loss A 12-year follow-up study. Medicine (United States) 95(44):e5312

25. Carlsson P-I et al (2015) Severe to profound hearing impairment: quality of life, psychosocial consequences and audiological rehabilitation. Disabil Rehabil 37(20):1849-1856

26. Turunen-Taheri S et al (2017) Patients with severe-to-profound hearing impairment and simultaneous severe vision impairment: a quality-of-life study. Acta Otolaryngol 137(3):279-285

27. Andersson G et al (2009) Tinnitus distress, anxiety, depression, and hearing problems among cochlear implant patients with tinnitus. J Am Acad Audiol 20(5):315-319

28. Kloostra FJJ et al (2015) Changes in tinnitus after cochlear implantation and its relation with psychological functioning. Audiol Neurotol 20(2):81-89

29. Stern AF (2014) The hospital anxiety and depression scale. Occup Med 64(5):393-394

30. Gomaa MAM et al (2014) Depression, anxiety and stress scale in patients with tinnitus and hearing loss. Eur Arch Otorhinolaryngol 271(8):2177-2184

31. Jayakody DM et al (2018) Association between speech and highfrequency hearing loss and depression, anxiety and stress in older adults. Maturitas 110:86-91

32. Guitar K et al (2013) Health effects of cochlear implants. N Zeal Med J 126(1375):9-26

33. Jones DA, Victor CR, Vetter NJ (1984) Hearing difficulty and its psychological implications for the elderly. J Epidemiol Community Health 38(1):75-78

34. Olze $\mathrm{H}$ et al (2011) Cochlear implantation has a positive influence on quality of life, tinnitus, and psychological comorbidity. Laryngoscope 121(10):2220-2227

35. Palmer CG et al (2013) Deaf genetic testing and psychological well-being in deaf adults. J Genet Counsel 22(4):492-507

36. Contrera KJ et al (2017) Association of hearing impairment and anxiety in older adults. J Aging Health 29(1):172-184

37. Helvik AS et al (2008) Renewed prescription of hearing aids: a clinically based study. Audiol Med 6(3):184-192

38. Vancampfort D et al (2017) The relationship between chronic physical conditions, multimorbidity and anxiety in the general population: a global perspective across 42 countries. Gen Hosp Psychiatry 45:1-6

39. Du Feu M, Fergusson K (2003) Sensory impairment and mental health. Adv Psychiatr Treat 9(2):95-103

40. Tyrer P (2018) Against the Stream: generalised anxiety disorder (GAD) — a redundant diagnosis. BJPsych Bull 42(2):69-71

41. National Institute for Health and Social Care Excellence (2014) Anxiety disorders quality standard [QS53] Webpage. Available at: https://www.nice.org.uk/guidance/cg113. Accessed 10 Dec 2018 